\title{
Netform Modeling and Applications
}

FRED GLOVER

DARWIN KLINGMAN

NANCY PHILLIPS
School of Business

University of Colorado

Boulder, Colorado 80309-0419

Graduate School of Business

The University of Texas

Austin, Texas 78712

Graduate School of Business

The University of Texas

Many real-world applications have profited from netform innovations in both modeling and solution strategies. Practical experience shows that advances in netform modeling and solution strategies overcome many of the difficulties in conceptual design and problem solving of previous approaches to system optimization. Moreover, they provide the type of technologies required of truly useful decision-planning tools, technologies that facilitate modeling, solution, and implementation. The ultimate test and worth of computer-based planning models, however, depends on their use by practitioners. In this tutorial, we show how certain algebraic models can be viewed graphically using netform modeling and describe several large practical problems we have solved. Some of our insights can make it easier for practitioners to take advantage of these technologies.

he growth of the computer industry
has profoundly influenced many areas, affecting none more dramatically than management science. Knowledge about ways to solve optimization problems in industry and government has exploded since World War II, largely because the computer can record and manipulate extremely large amounts of data. Without this capability, many of the tools of management science would be mere theoretical niceties.

The techniques for building, solving, refining, and analyzing computer-based 
planning models have evolved steadily as computer hardware has changed. This evolution has spawned two new and important technologies, computer implementation technology and problem representation technology. Both have been strongly affected by developments in the field of optimization. Within the optimization field, perhaps no domain has had a greater impact, as the source of innovations in implementation and representation technology, than network optimization. These innovations are changing our notions about how

\section{Knowledge about ways to solve optimization problems has exploded since World War II.}

to conceptualize and exploit optimization problems. The technologies of computer implementation and problem representation have profited from network optimization chiefly because advances in this field have intimately related problem solving to the identification and exploitation of structure. Over the last decade and a half, the development of models based on characterizing structure for the purpose of insight and more effective solution has resulted in the field of netforms-or network-related formulations. This field has (1) expanded awareness among OR theoreticians and practitioners of the range of problems that can be formulated as networks and generalized networks; (2) identified ways to express problems-and key components of problems -in a network format, especially in contexts that do not immediately suggest this possibility; (3) introduced flexible notations to identify additional constraining conditions, such as "all-or-none" and "multiple choice" flow restrictions (and integer restrictions in generalized networks), which make it possible to represent problems outside the usual network category in a network-related framework; (4) developed a repertoire of model constructs to document which netforms are best for communication and which are best for solution (not always the same); and (5) evolved computer implementation strategies to take fullest advantage of netform representations.

The fertile interaction between computer implementation technology and problem representation technology associated with these developments has greatly profited from research on pure and generalized network problems, subsequently adapted to broader contexts. These innovations include those of Barr, Glover, and Klingman [1977, 1979]; Bertsekas and Tseng [1988]; Bradley, Brown, and Graves [1977]; Cunningham [1979]; Galil and Tardos [1986]; Gilsinn and Witzgall [1973]; Glover, Karney, and Klingman [1972, 1974]; Glover, Karney, Klingman, and Napier [1974]; Glover and Klingman [1988b]; Glover, Klingman, and Phillips [1988]; Glover, Klingman, and Stutz [1974]; Helgason, Kennington, and Lall [1976]; Hung [1983]; Ikura and Nemhauser [1986]; Jensen and Barnes [1980]; Karney and Klingman [1976]; Kennington and Helgason [1980]; Orlin [1984, 1988]; Ross and Soland [1975]; Srinivasan and Thompson [1972, 1973]; and Tardos [1985]. This research has dramatically reduced the cost of solving problems in the linear and mixed integer network domain, 
beyond the reductions due to improvements in computer hardware or software. For example, the cost of solving network problems with 2,400 equations and 500,000 arcs has been reduced from thousands of dollars in the late ' 60 s to less than $\$ 100$ in the late ' 80 s.

Using specialized network algorithms to solve network problems provides significant gains over using commercial linear programming (LP) codes. The best solution algorithm for pure network problems in the late ' 60 s was the out-of-kilter algorithm [Dantzig 1963; Ford and Fulkerson 1962]. Testing in the late '60s through the '70s, Glover, Karney, and Klingman [1974]; Glover, Karney, Klingman, and Napier [1974]; Karney and Klingman [1976]; and Srinivasan and Thompson [1973], showed

\section{The cost of solving network} problems with 2,400 equations and 500,000 arcs has been reduced from thousands of dollars in the late '60s to less than $\$ 100$ in the late ' 80 s.

that the primal simplex algorithm was best if one devised specialized data structures and pivot and start procedures. In the ' 80 s, the testing of nonextreme point algorithms [Bertsekas and Tseng 1988; Glover and Klingman 1988b] began to challenge the primal simplex algorithm; it is becoming less clear which algorithm is best for pure network problems. For generalized network problems, the primal simplex algorithm with specialized data structures is still fastest.
These advances in solution technology have stimulated the development of modeling techniques for handling a multitude of problems that arise in applications of scheduling, routing, resource allocation, production, inventory management, facilities location, distribution planning, and other areas. These new modeling techniques [Glover, Hultz, and Klingman 1978, 1979; Glover and Klingman 1977; Glover, Klingman, and McMillan 1977; Glover and Mulvey 1980] are mathematically and symbolically linked to network and augmented network structures and constitute the central focus of netform technology. This technology allows users to conceptualize formulations of their problems graphically. Its pictorial aspect has proven to be extremely valuable in communicating and refining problem interrelationships without the use of mathematics and computer jargon. Thus it protects the nontechnical person against technical legerdemain and exaggerated claims of model "realism." This technology also often yields a model that can be solved as a sequence of linear network problems or by merging solutions to linear networks in progressively refined stages.

\section{Pure Network Models}

Pure network problems embody a group of distinct model types, including shortest path, assignment, transportation, and transshipment problems. Any of these network problems can be characterized by a coefficient matrix that has at most one +1 and one -1 entry in each column. The most general of these model types is the transshipment problem.

The transshipment model appears in many applications, either directly or as a 
subproblem. An illustration of this model for a cash-flow problem is depicted in Figure 1 . The arrows in network models are called arcs and the circles are called nodes. Triangles leading into or out of nodes represent supplies and demands, respectively. Arcs indicate allowable flow paths between nodes and have lower and upper bounds, shown in parentheses, and costs, shown in rectangles.

The objective in the transshipment problem is to determine how much to ship along each arc within the limits stipulated by the bounds in order to satisfy all supplies and demands and to minimize total cost. By satisfying supplies and demands we mean that the total flow into the node minus the total flow out must equal its demand, and the total flow out of the node minus the total flow in must equal supply. For all other nodes, the flow into the node must equal the flow out.

It is important to understand how a transshipment problem may be stated mathematically to appreciate the connections between graphical and algebraic structures. To state a network problem algebraically, we define a variable for each arc. For example, let $X_{i j}$ denote the flow on the arc from node $i$ to node $j$ and $c_{i j}$ denote the unit cost on this arc. Henceforth, an arc will be denoted as an ordered pair $(i, j)$ where the first component specifies the from node and the other component the to node. Next we create the objective function for the problem as an expression involving the costs and variables. For the problem in Figure 1, the objective function would be

$2 X_{A C}+3 X_{A D}+5 X_{B D}+2 X_{B E}+4 X_{C D}+3 X_{C F}$ $+10 X_{C G}+5 X_{D C}+3 X_{D G}+2 X_{E G}$.
Upon identifying the objective function, we create a constraint for each node that expresses the restriction on flow into and out of the node. To do this, it is convenient to view a supply as an inflow and a demand as an outflow. Then the requirement at each node can be expressed as Total Inflow $=$ Total Outflow, or equivalently, Total Inflow - Total Outflow $=0$.

The customary transposition of any constant term of this equation to the right hand side causes supplies to be denoted as negative quantities and demands to be denoted as positive quantities. To see this, consider constructing the "Total Inflow - Total Outflow $=0$ " equation for node $C$ in Figure 1. The total flow into node $C$ consists of the five units of supply and $\left(X_{A C}+X_{D C}\right)$. The total flow out of node $C$ is $X_{C F}+X_{C G}+X_{C D}$. Thus we have $5+X_{A C}$ $+X_{D C}-\left(X_{C F}+X_{C G}+X_{C D}\right)=0$, or after transposing the constant term, $X_{A C}+X_{D C}$ $-X_{C F}-X_{C G}-X_{C D}=-5$. Thus the supply of five becomes expressed as a negative quantity because of its movement to the right hand side of the equality sign. On the other hand, a demand, (which is included in the outflow that is subtracted in the "inflow-outflow" equation), appears as a positive quantity when moved to the right hand side. This also discloses, incidentally, that a supply may be viewed as a negative demand, and vice versa. The entire algebraic statement of the capacitated transshipment cash flow problem is also shown in Figure 1.

Each $X_{i j}$ appears in exactly two node equations, (the equation for node $i$ and the equation for node $j$ ). Further, since $X_{i j}$ contributes to the outflow of the node $i$ equation and to the inflow of the node $j$ equa- 


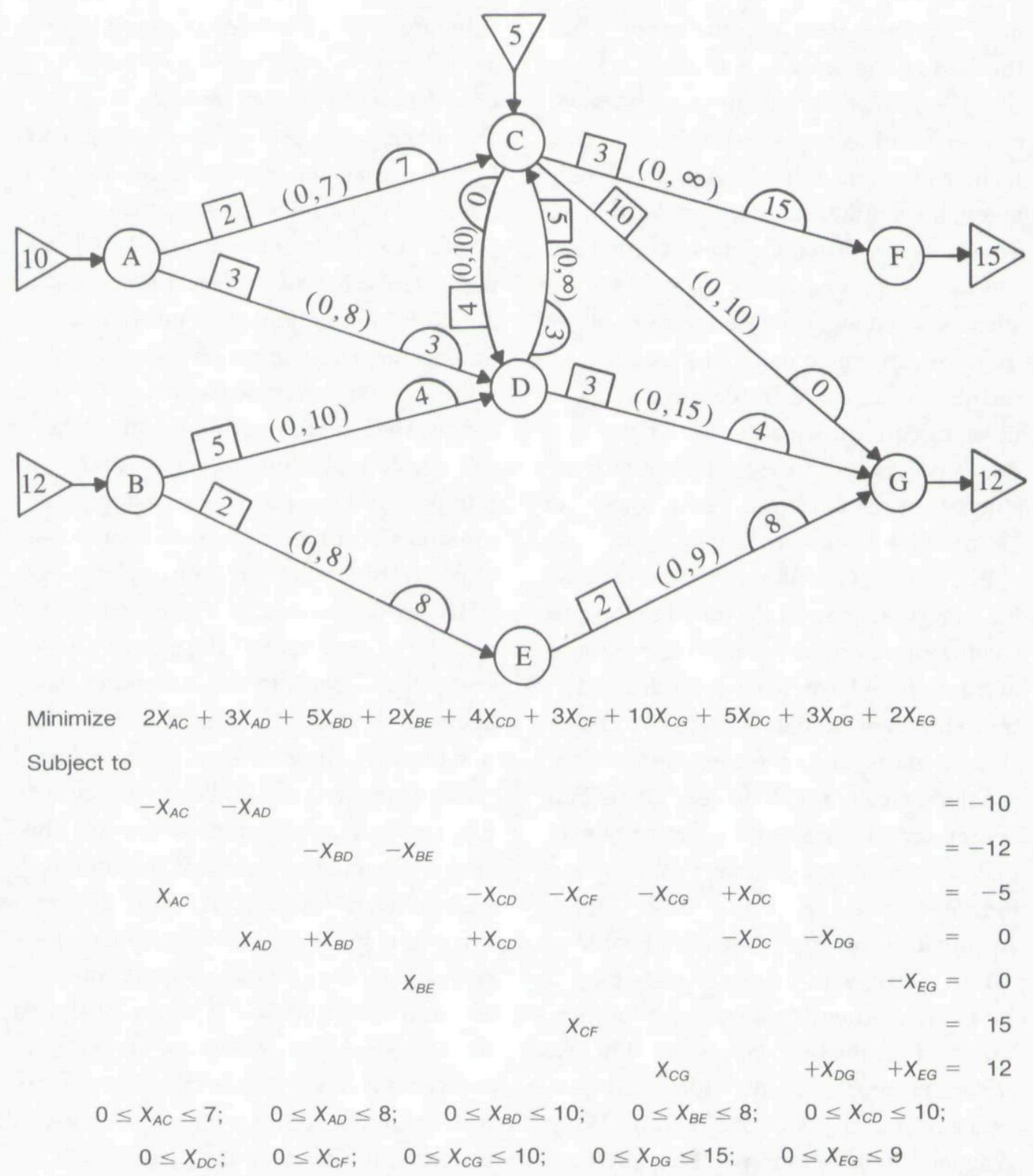

Figure 1: Capacitated transshipment cash-flow model. The nodes correspond to subsidiaries of a central company that operates in different locations. The supplies and demands represent excess or deficit cash, respectively. Thus, nodes $A, B$, and $C$ have excess funds, nodes $D$ and $E$ have no funds, and nodes $F$ and $G$ have deficit funds.

The arc from node $A$ to node $C$ indicates that it is possible to transfer funds from subsidiary $A$ to subsidiary $C$. The absence of an arc indicates that it is not possible to transfer funds directly between the corresponding pair of subsidiaries (though it may be possible to transfer funds indirectly by means of a sequence of arcs through intermediate subsidiaries). The arc from node $A$ to node $C$ has a lower bound of 0 , an upper bound of 7 , and a cost of 2 . The numbers in the semi-circles on the arcs illustrate a solution which satisfies the node equations and the bound requirements. 
tion, it appears with a coefficient of -1 in the former and with a coefficient of +1 in the latter. Thus, each column of the coefficient matrix has one -1 and one +1 entry. If the restrictions at the nodes are stated as inequalities rather than equations (that is, Total Inflow - Total Outflow $\leq 0$ or Total Inflow - Total Outflow $\geq 0$ ), then slack or surplus variables are added to convert these constraints to equations, and it is admissible for columns to contain a single nonzero entry. Inequality restrictions at nodes generally arise by stipulating that supplies or demands must be "at least" or "at most" a certain amount.

By reversing the above steps, it is possible to represent any LP problem whose coefficient matrix has at most one +1 and at most one -1 entry per column as a transshipment problem. That is, to construct a graph that corresponds to such a problem, create a node for each constraint and an arc for each variable, affixing supplies, demands and bounds in the manner indicated.

\section{Applications of Pure Network Problems}

Pure network problems provide models for many mathematical optimization problems and for major components of many additional problems. Inventory maintenance problems [Evans 1978; Taha 1971; Wagner 1969] for example, typically exhibit an underlying network structure. A cousin of the inventory maintenance problem is the PERT/CPM problem, which seeks the best way to sequence a complex set of interdependent activities. The PERT/ CPM framework, which constitutes one of the simplest network model forms, has been used in a variety of practical applications (including construction of the Polaris submarine) and has been reported to save substantial costs and greatly speed completion of complex projects.

Other types of problems involving the effective management of resources also frequently exhibit network structures. Such problems are becoming increasingly important in government and industry. Direct network formulations of water resource management problems, for example, are finding use in a number of states. In these, canals, river reaches, and pipelines take the role of arcs, while reservoirs and pumping stations take the role of nodes. Planning over time frequently looms as a major consideration in these applications.

The Texas Water Development Board and the government of Poland introduce what-if analyses into water resource management by using a succession of simulations having alternative supply and demand configurations and solve the resulting network for each simulation run. The step of finding the optimum solution to each network problem is used to determine the best response to meet demands for water use, given a particular supply-anddemand configuration. (This use of simulation, in which parameters are varied to achieve what-if analyses by means of rigorous solution techniques, is to be contrasted with the common "quasioptimization" use of simulation.) To analyze the full range of relevant configurations, roughly 500 such runs must be made each month. The feasibility and costeffectiveness of such runs is due to the efficiency with which the underlying networks are solved.

The problem of determining flows and heads in a general pipeline system (such as 
in municipal water systems) with reservoirs, pumps, gate and check valves, given fixed inputs and withdrawals has been shown [Collins et al. 1978] to be equivalent to a convex transshipment problem under the assumption of convex head losses. Such problems are easily solved as ordinary transshipment problems using a piecewise linear approximation of the convex function. Since the convexity requirements are usually satisfied for real pipe networks, this is an example of another class of real-world problems that can now be handled by network procedures with far greater effectiveness than by the procedures used in the past.

Another important instance of the use of network models occurs in manpower promotion and assignment problems. AT\&T has developed such models to guarantee acceptable hiring and promotion policies in accordance with HEW rules and regulations. A number of cash-management problems can also be modeled as transshipment problems. These models include sources of funds in addition to cash (such as maturing accounts and notes receivable, sales of securities, and borrowing) and uses of funds other than a single investment. The generalized network model we will discuss later makes it possible to further incorporate discount, interest, and other financial considerations directly into the model.

Many nonlinear problems involve network subproblems. One of the most basic and prevalent forms of nonlinear problems is the fixed-charge network problem, whose major offshoots include the genre known as location problems. The nonlinear element of a fixed-charge network is the fixed-charge arc, which has the following special property: whenever the arc is "used" (that is, permitted to transmit flow), a charge is incurred that is independent of the amount of flow across the arc. Fixed-charge networks have been used to model problems of plant and warehouse location, equipment purchasing and leasing, personnel hiring, and offshore oildrilling platform location, among others [Taha 1971; Wagner 1969].

To better utilize forecasting data and to support economically rational operational decisions, Klingman, Phillips, Steiger, Wirth, Padman, and Krishnan [1987] and Klingman, Phillips, Steiger, Wirth, and Young [1986] developed an optimizationbased decision support system for planning supply, distribution, and marketing, called the SDM system, for Citgo Petroleum Corporation. The SDM system integrates Citgo's key economic and physical supply, distribution, and marketing characteristics over a short-term (11-week) planning horizon. This time horizon incorporates inventory planning and time lags for manufacturing and distribution. To model this timing problem, they partitioned the basic model into time zones and employed replications of the basic model to accommodate the distinct time periods (weeks), as shown in Figure 2.

Top management uses the system to make such decisions as where to sell products, what price to charge, where to buy or trade products, how much to buy or trade, how much of a product to hold in inventory, and how much product to ship by each mode of transportation. All information is provided by location, by line of business, and by week. The model incor- 


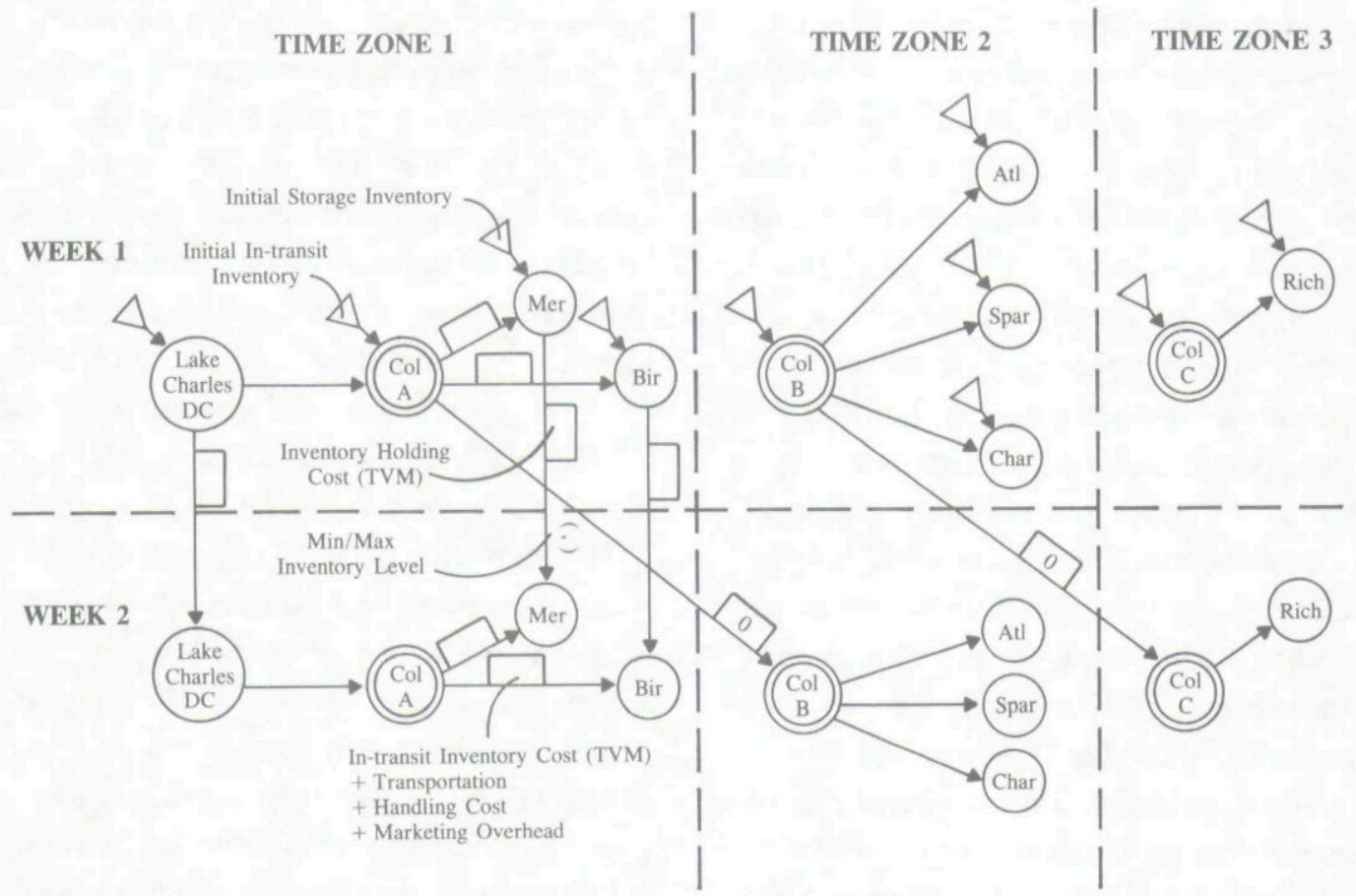

Figure 2: Network representation of time zones and time periods. Single circles represent product storage terminals, specifically a distribution center terminal at Lake Charles and other terminals at Meridian, Birmingham, Atlanta, Spartanburg, Charlotte, and Richmond. Double circles represent locations in a distribution pipeline called Colonial in each time zone. For example, "Col A" represents the segment of the Colonial pipeline in time zone 1 which contains the product storage terminals that are within one week's travel time from the refinery. "Col B" represents the segment of the Colonial pipeline in time zone 2 which contains those terminals between one and two weeks away, etc. (Double circles are used for pipeline nodes to make the network easier to "read." Due to the large size of the problem and the number of different components which nodes were used to represent, the network diagram for the entire problem also employs additional node symbols, such as half circles and ellipses.) The vertical arcs between product storage terminal nodes represent inventory held at storage terminals between time periods. The diagonal arcs between pipeline nodes represent in-transit inventory. For example, product which is in the " $\mathrm{A}$ " segment of the Colonial pipeline in week 1 will travel to the "B" segment in week 2 (if it is not lifted into the Meridian or Birmingham terminals). Thus week 1 demand in time zones 2 and 3 can only be satisfied by initial in-transit or storage inventory, modeled by the supply triangles shown, since product cannot travel from Lake Charles to Col B or Col C in one week. (Demand triangles, not shown, would be attached to each product terminal node.)

porates the critical timing considerations associated with all of these decisions.

The SDM system contains a separate minimum cost (or as an option, maximum profit) flow network model for each product (corresponding to the product managers who work with the supply, distribu- tion, and marketing personnel associated with each product). Each model is generated using input data from a corporate data base, and the optimal solution is aggregated into output reports tailored for use by the individual operational managers. The objective function for the profit 
maximization model is calculated as total sales revenue less expenses, such as transportation costs, terminal handling costs, and the time value of money associated with in-transit and terminal inventory. The constraints of the model include forecasted price and demand functions, inventory capacities, exchange agreement options, spot market purchase and sale volume limits, purchase, sale, and trade agreement options, and retail demand. Management can also include preliminary shipment schedules in the model as finalized decisions. Finally, the SDM system employs rule-based artificial intelligence systems to arrive at exchange agreement payback rules for model constraints, and it uses an expert system approach to screen output reports to provide management with exception reports.

Due to the complex combination of multiple product sources, distribution timing, trading alternatives, and price sensitive demands, the authors used a number of modeling techniques to control the size of the model and to keep the model in network format and linear. These greatly extended the model shown in Figure 2. The network framework was important for two main reasons. First, the pictorial aspect of network models was extremely valuable to the authors in working with a broad spectrum of personnel to develop, validate, and implement this integrated model. Second, network models permit rapid solution speed, which allowed management to respond quickly to the dynamics of a commodity market industry by using the SDM system extensively in what-if sessions. For example, on a medium-sized computer, an IBM 4381 mod 2, each product model con- sisting of approximately 3,000 equations and 15,000 variables can be solved using an efficient network optimization code [Glover and Klingman 1982] in approximately 30 seconds. (To run the entire SDM system, model generation requires about two minutes and report processing about seven minutes.) The SDM system allowed Citgo to reduce product inventories by $\$ 116$ million (based on historical inventory-to-sales ratios) resulting in a \$14 million per year reduction in working capital costs. The system also provided many insights into supply, distribution, and marketing that contributed an estimated additional $\$ 2.5$ million per year to bottom line profits.

In another application of pure network transshipment modeling, Klingman and Phillips [1984] developed a new modeling and solution approach to make enlisted personnel assignment decisions for the Marine Corps. Our military services seek to accommodate three primary types of goals or criteria in assigning enlisted personnel to positions. Each goal type may have several subgoals, leading to a model that has many criteria or objective functions. These criteria express the desire of the military to fill as many positions as possible in some priority fashion, the cost of assigning a person to a position, the utility of such an assignment to the military organization, and the desirability of the assignment to that person. In addition, the Marine Corps also specifies proportionate or disproportionate percentage fill policies for handling shortages within a job type. The criteria are preemptive and are handled in order of decreasing importance of the three goal types: maximum fill, maximum priority 
distribution fill, and maximum fit.

To evaluate alternative modeling and solution approaches to the Marine Corps' problem, Klingman and Phillips used a random problem parameter generator provided by the USMC to generate a single test problem. The generator produced a problem that had 10,000 persons, 240 job types, and three job groups. Two of the groups have proportional fill criteria and the other a disproportional distribution fill criteria. The highest priority group requires $5 i$ persons to fill its $i$ th job type and the other groups require a constant number of persons to fill their job types. The parameter generator randomly generates eligible person/job arcs and associates four fit criteria coefficients with each one. Using this parameter generator, Klingman and Phillips developed a large-scale multicriteria pure network transshipment model with 10,245 nodes, 748,930 arcs, and 11 criteria. (There are four fit criteria, three priority-fill criteria-two proportional and one disproportional, three job-group-fill criteria, and an overall fill criterion to maximize the total number of people assigned to jobs.) The model correctly handles the proportionate and disproportionate percentage fill policies and simultaneously yields integer solutions for large-scale problems. Klingman and Phillips solved the problem using six different weighting strategies for aggregating the 11 criteria in the model. The best of these strategies required about 127 minutes of CPU time on a PRIME 550 mini-computer. It is significant that this large-scale multicriteria test problem could be solved on such a lowcost computer (less than $\$ 200,000$ in 1980) in a reasonable period of time.

\section{Generalized Networks}

The generalized network (GN) problem represents a class of LP problems that has received much less attention than it deserves. Recently, as many new generalized network applications have been identified and computer codes able to solve these problems efficiently have emerged, generalized networks are coming to be appreciated as rivaling or even surpassing pure networks in their practical significance. Generalized networks include pure networks as a special case. The GN problem, by allowing nonzero column entries other than \pm 1 , is actually the broadest classification of linear network-related problems. GN problems arise in resource allocation, production, distribution, scheduling, capital budgeting, and other settings.

The most effective procedures for modeling and communicating pure network problems are based on viewing these problems as directed graphs. Generalized network problems can also be represented as directed graphs by means of appropriate conventions. In particular, the coefficient matrix can be transformed (if the variables are bounded) so that at least one entry in any column with two nonzero entries is -1 . In this way, a directed arc is created that leads from the node associated with the -1 to the node associated with the other nonzero entry. (If both entries are -1 , the arc may be directed either way.) A single nonzero entry in a column is represented by an arc that touches only one node.

There is an important distinction between arcs in pure network problems and arcs in GN problems. An arc of a generalized network has a multiplier. This multi- 
plier is the nonzero coefficient associated with the node at the arrowhead of the arc (that is, the terminal node of the directed arc). In pure networks, this multiplier is always +1 .

These ideas are illustrated by the GN problem shown in Figure 3 along with the associated network. As with pure network problems, each row of the coefficient matrix is associated with a node and each column with an arc. That is, a node corresponds to a problem equation and an arc corresponds to a problem variable. Consequently, each arc has a cost, lower bound, and upper bound. Costs are shown within rectangles, and bounds are shown within parentheses. Arc multipliers are shown within triangles.

The multiplier of a generalized network problem acts upon the flow across an arc; the amount of flow starting out on the arc will not necessarily be the amount arriving at the opposite end. Specifically, the flow entering the arc is multiplied by the value of the multiplier to produce the quantity of flow leaving the arc. The arc's cost, lower bound, and upper bound refer only to the units of flow entering the arc.

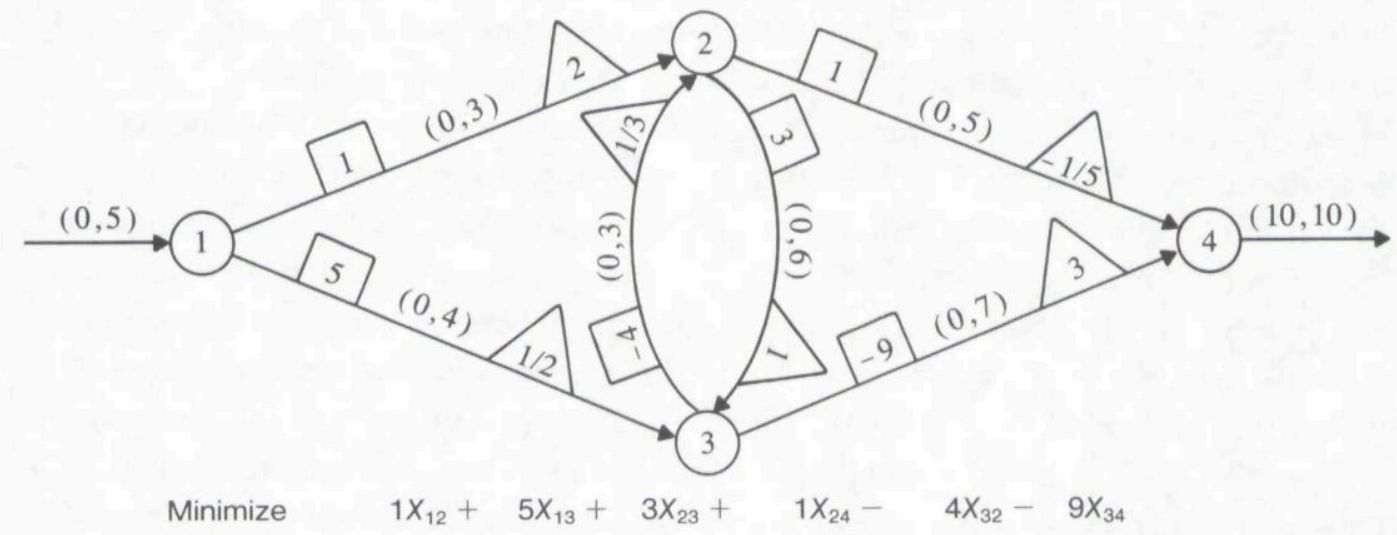

Subject to

$$
\begin{array}{rlrrr}
X_{1}-1 X_{12} & -1 X_{13} & & \\
2 X_{12} & & -1 x_{23} & -1 X_{24}+1 / 3 X_{32} & =0 \\
& 1 / 2 X_{13}+1 X_{23} & -1 X_{32}-1 X_{34} & =0 \\
& & -1 / 5 X_{24} & +3 X_{34}-X_{4}=0 \\
0 \leq X_{1} \leq 5, & 0 \leq X_{12} \leq 3, & 0 \leq X_{13} \leq 4, & 0 \leq X_{23} \leq 6, & \\
0 \leq X_{24} \leq 5, & 0 \leq X_{32} \leq 3, & 0 \leq X_{34} \leq 7, & 10 \leq X_{4} \leq 10 &
\end{array}
$$

Figure 3: Generalized network. Supply into node 1 and demand out of node 4 are modeled using bounded arcs, rather than supply and demand triangles. Thus node 1 has a supply of at most 5 while node 4 has a demand of exactly 10 . (The arc out of node 4 could have been modeled alternatively as a demand triangle with a demand of 10 . In that case the variable $X_{4}$ would be omitted, and the last equation would be $-1 / 5 X_{24}+3 X_{34}=10$.) If 2 units start on the arc from node 1 to node 2 , the multiplier of 2 will cause 4 units to arrive at node 2 . Likewise, 10 units starting on the arc from node 2 to node 4 will result in -2 units arriving at node 4 (or, equivalently, 2 units leaving node 4 on that arc) since the multiplier in this case is $-1 / 5$. 


\section{Applications of Generalized Networks}

Generalized networks can successfully model many problems that have no pure network equivalent. This is made possible by two useful interpretations of arc multipliers. First, multipliers can be viewed as modifying the amount of flow of some particular item. By means of flow modification, generalized networks can model such situations as evaporation, seepage, deterioration, breeding, interest rates, sewage treatment, purification processes with varying efficiencies, machine efficiency, and structural strength design. However, it is also possible to interpret the multiplication process as transforming one type of item into another. This interpretation provides a way to model such processes as manufacturing, production, fuel to energy conversion, blending, crew scheduling, manpower to job requirements, and currency exchanges. The following applications are examples of possible uses of generalized networks.

Bhaumik [1973] modeled a water distribution system with losses as a generalized network problem. This model is primarily concerned with the movement of water through canals to various reservoirs. However, it also considers the retention of water over several time periods. The multipliers in this case represent the losses from evaporation and seepage.

Gilliam and Turner [1974] proposed a file reduction model that has the form of a generalized transportation model with a single extra constraint. This model is designed to facilitate the reduction of extremely large microdata files to smaller, statistically representative files. The objective is to minimize the amount of informa- tion lost in the reduction process. The arcs represent paths from the original records to the reduced records. A nonzero flow on an arc implies that the original record is to be represented by the reduced record connected to it by that arc. The multipliers on the arcs are used to insure that the reduced file is truly representative of all of the original records.

Kim [1972] utilized generalized networks to represent copper refining processes and modeled the electrolytic refining procedure by a large d-c electrical network. The arcs are current paths with the multipliers representing the appropriate resistances. In this way, Kim analyzes the effect of short circuits in the refining process.

Charnes and Cooper [1961] identified applications of generalized networks for both plastic-limit analysis and warehouse funds-flow models. In plastic-limit analysis, the network is generated by forming the equations for horizontal and vertical equilibria and by employing a coupling technique. The warehouse funds-flow model is actually a multi-time period model. The arcs are used to represent sales, production, and inventory of both products and cash. The multipliers are introduced to facilitate the conversions between cash and products.

Crum [1976] modeled a cash management problem for a multi-national firm as a generalized network. This model incorporated transfer pricing, receivables and payables, collections, dividend payments, interest payments, royalties, and management fees. The arcs represented possible cash-flow patterns and the multipliers, costs, savings, liquidity changes, and exchange rates. 
While the Alaskan Pipeline was being built, Congress funded the development of a model for the distribution of natural gas across the United States. The model includes the largest 240 distributors and the largest 100 pipelines in the United States. The model used multiple objective functions to represent the hierarchical way gas is distributed (residential customers before commercial customers, and so forth). Using a commercial LP code to solve this model cost over $\$ 1,000$ for each solution run.

However the problem can be modeled as a generalized network; because gas in the pipeline is used to drive the pipeline pumps, gas is lost as it moves along the pipeline. The generalized network code NET-G [Glover, Hultz, Klingman, and Stutz 1978] solved the model for about $\$ 12$ per run.

Glover, Glover, and Martinson [1984] use a netform model somewhat differently; they formulated a large-scale linearprogramming problem for resource planning as a generalized network with side constraints. These constraints had the special form of compelling flows on certain arcs to satisfy proportionality restrictions based on flows of other related arcs. Significantly, all linear programming problems can be represented as generalized networks with these types of side constraints, although in this setting these constraints arose naturally and were not created as a modeling device.

The goal of this model was to determine the optimal management of public rangelands by the US Bureau of Land Management (BLM) relative to uses for grazing and for supporting wildlife. This required identifying optimal numbers of animals of various types, in consideration of their dietary requirements, that would be allowed to populate specific regions. The large size of the problem arose from the massive area of rangelands involved and the multiple nutritional and geographic factors affecting the possible allocations of different wildlife types to these regions. A simplified component of the model is shown in Figure 4.

To carry out necessary analysis, the BLM wanted to solve multiple versions of these problems daily. The amount of time needed to solve them using standard linear programming made it impractical. A specialized procedure for progressively setting and revising arc bounds in the netform, however, made it possible to solve these problems 20 to 300 times faster than by the previous LP system, obtaining solutions within 95 percent of optimality (usually 98 percent or better). The speed improvements were greatest for the larger problems where the time factors were increasingly critical. The Bureau of Land Management routinely uses the netformbased system an average of 700 times a month throughout the US.

Another generalized transshipment network was the basic component of an optimization-based logistics planning system that Klingman, Mote, and Phillips [1988] developed for W. R. Grace Company, one of the nation's largest suppliers of phosphate-based chemical products. The mathematical model underlying this system includes production, distribution, multiple time periods, and multiple commodities. W. R. Grace initially formulated the model as a linear programming problem with 3,696 constraints and 21,564 


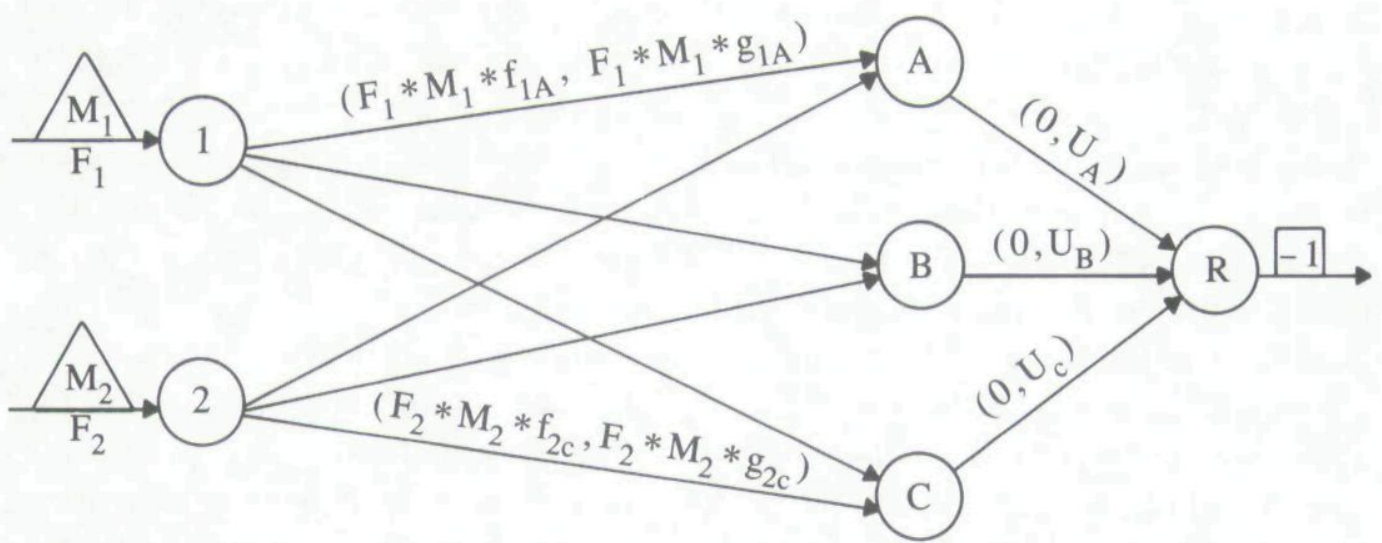

Figure 4: US Bureau of Land Management model. In the geographical region represented by this diagram, there are two types of animals, represented by the nodes labeled 1 and 2, each of which can consume varying amounts of three types of plants, represented by the nodes labeled A, B and C. The volume of food consumed by each animal of types 1 and 2 are represented by the multipliers $M_{1}$ and $M_{2}$, respectively, shown associated with the arcs entering nodes 1 and 2. These multipliers transform numbers of animals flowing on these arcs, denoted by $F_{1}$ and $F_{2}$ and determined by allocations in other parts of the network, into total volume of food consumed. Each of the arcs from the animal nodes to the plant nodes has a lower and upper bound of the special form illustrated within parentheses for the first and last of these arcs. The distinguishing feature of these bounds is that they are variable, and depend on the amounts of flow $F_{1}$ and $F_{2}$ entering nodes 1 and 2 . Specifically, two fractions $f_{1 A}$ and $g_{1 A}$ are applicable to the arc from node 1 to node $A$, indicating that the amount of flow on this arc must be at least $F_{1} * M_{1} * f_{1 A}$ and at most $F_{1} * M_{1} * g_{1 A}$ (which identifies the least and greatest quantities of plant $\mathbf{A}$ that type 1 animals will consume in an appropriate diet). Similar variable bounds bracket the flows on the other arcs from animal nodes to plant nodes, as illustrated on the arc from node 2 to node $\mathrm{C}$. The total available quantity of each of the three plant types in the given region is represented by the bounds on the arcs out of nodes A, B and C. The BLM objective was to utilize the total plant food available to the fullest extent possible, subject to the dietary requirements of the various animal species. This was accomplished by placing a cost of -1 (that is, a profit of 1 ) on the arc leaving node $R$ and using a cost-minimizing algorithm to solve the problem.

variables. An integrated approach to modeling and solving the problem enhanced top management's understanding of the model and made the problem more tractable for the company's DEC 20/60 computer. The key features of this approach are decomposition of the problem into a generalized network component and a small linear nonnetwork component, transformation of the generalized network component into a pure network component, incorporation of most of the nonnetwork component into the pure network via an innovative relaxation approach, and incorporation of the remainder into the objective function via Lagrangian procedures. The resulting model relaxation was solved using efficient pure network solution techniques to obtain an advanced starting basis for a basis partitioning algorithm. This approach reduced solution time approximately 10 -fold. In addition, W. R. Grace 
used insights obtained from the solutions to make multimillion dollar decisions.

Additional applications of generalized networks include machine loading problems [Charnes and Cooper 1961; Wagner 1969], blending problems [Charnes and Cooper 1961; Wagner 1969], the caterer problem [Dantzig 1963; Wagner 1969], and scheduling problems such as production and distribution problems, crew scheduling, aircraft scheduling, and manpower training [Charnes and Cooper 1961; Dantzig 1963; Wagner 1969].

\section{Integer Generalized Networks}

An especially important realm of application extends the use of generalized networks by requiring that flows on particular generalized arcs must occur in integer (whole number) amounts. Introducing the integer requirement into the $\mathrm{GN}$ problem enables it to model an unexpected diversity of additional applications, including such problems as scheduling variable-length television commercials into time slots, assigning jobs to computers in computer networks, scheduling payments on accounts where contractual agreements specify lump sum payments, and designing communication networks with capacity constraints.

Using integer requirements in GN problems enables any $0-1 \mathrm{LP}$ problem to be modeled as an integer GN problem [Glover, Klingman, and McMillan 1977; Glover and Mulvey 1980]. These techniques can also accommodate mixed integer 0-1 LP problems where the continuous part of the problem is a transportation, transshipment, or generalized network problem itself. An illustration in Crum, Klingman, and Tavis [1979] shows how contemporary financial capital allocation models can be modeled as integer GN problems. Important real-world applications with such a "mixed" structure also include a variety of plant location models, energy models, and physical distribution models.

Integer generalized networks are also used to model requirements that flows on certain arcs must equal their upper or lower bounds, or that at least (or at most) a specified number of arcs from particular sets must have zero flows and other similar logical requirements. These netform representations consisting of networks with easily specified side conditions are able to rigorously express all problem elements that would ordinarily require expression in an algebraic form (as by customary mathematical programming formulation techniques). Consequently, they effectively replace the obscure and unilluminating algebraic representation by an equivalent, but much easier to understand, pictorial representation. We will show how this comes about by several examples, beginning with a fundamental technique that reappears in various guises in a variety of practical settings. The applications also demonstrate that the underlying network-related structures of the netform approach can often be exploited by special solution methods that are far more efficient than the methods previously developed for the algebraic representations.

Figure 5 illustrates a useful modeling device based on integer constrained generalized arcs commonly employed in the netform approach. An extension of this device to handle an even more useful set of conditions is illustrated in the model compo- 
nent shown in Figure 6. The combination of arc multipliers and 0-1 integer restrictions gives rise to what generally is called an integer network or a 0-1 generalized network. The following real-world applications demonstrate more fully uses of this netform modeling tool.

\section{Applications of Integer Generalized}

\section{Networks}

The netform concept has also improved the solution of a mixed integer programming problem for determining the minimum cost refueling schedule for nuclear reactors. Kazmersky [1974] initially modeled this problem as a mixed integer pro-

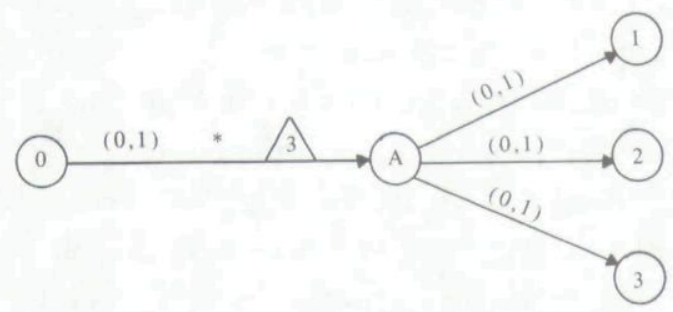

Figure 5: Generalized network with integer flow restriction. This figure represents only a component of a model, and thus no supplies or demands are shown. The bounds, costs, and multipliers are depicted by the same conventions employed before. In addition, the asterisk on the arc from node 0 to node $\mathrm{A}$ indicates that its flow must be an integer. If the flow is 0 , then $3 \cdot 0=0$ and no flow gets transmitted to node $A$. But if the flow is 1 , then 3 units are transmitted to node A. Further, because of the upper bounds of 1 on each of the three arcs leaving node $A$, the only possible way to distribute the three units flowing into node $A$ is to send exactly one unit to each of the nodes 1,2 , and 3 . Thus, by giving all arcs bounds of 0 and 1 and introducing a generalized arc, the following effect has been achieved: when the flow on the arc from node 0 to node $\mathbf{A}$ is 0 , the flow on each of the three arcs out of node $\mathbf{A}$ is $\mathbf{0}$; when the flow on the arc from node 0 to $A$ is 1 , the flow on each of the three arcs out of node $A$ is 1 . gramming problem with no apparent connection to networks. However, after working closely with Dr. Kazmersky, we discovered a way to express the problem by a $0-1 \mathrm{GN}$ that was not only equivalent to the original formulation but that also succeeded in reducing the size of the problem [Glover, Klingman, and Phillips 1988].

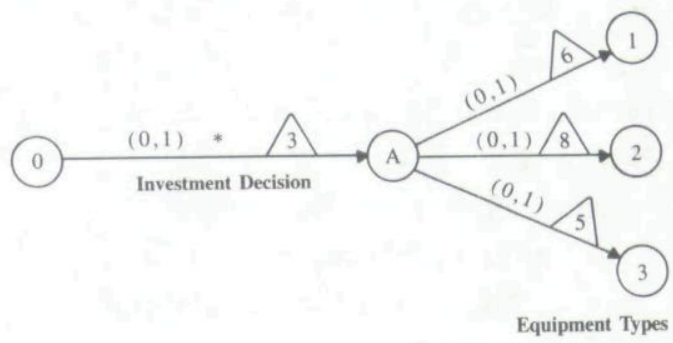

Figure 6: An equipment investment. This figure is the same as Figure 5 except that multipliers have now been added to the three arcs leaving node A. For concreteness, we may suppose this diagram represents an investment decision: to invest in project $A$ (if the flow on the arc from 0 to $A$ is 1 ) or not to invest in project $A$ (if the flow on the arc from 0 to $A$ is 0 ). Then the nodes 1,2 , and 3 identify different components of this investment project. In this example, the investment project is set in an equipment purchase context, and nodes 1, 2, and 3 represent different equipment types. In other contexts, these nodes might represent different types of aircraft in a fleet, different parcels of land in a real estate venture, different types of stock in a portfolio, etc. The multipliers on the arcs into nodes 1,2 , and 3 represent the quantities of each component of project $A$ (each type of equipment) that would be acquired if in fact the decision is made to invest in that project. By the conventions and flow relationships previously described, the diagram of Figure 6 transforms the investment into its components in precisely the manner desired; that is, a flow of 1 on the arc from node 0 to node $A$ (representing the decision to invest) translates into six units of equipment 1 at node 1 , eight units of equipment 2 at node 2 , and five units of equipment 3 at node 3 . 
While the original formulation by itself consumes more than 20 pages [Kazmersky 1974], we described the full 0-1 GN model in a handful of pages without using complex mathematical notation.

In addition, making use of the $0-1 \mathrm{GN}$ formulation, we were able to develop a branch-and-bound solution procedure that solved GN subproblems, yielding significant gains over previous solution efforts. We solved four versions of this problem using data supplied by the Tennessee Valley Authority. The first three versions, which required half an hour to two hours to solve on an IBM 4381 computer using the MPSX solution system, took less than 20 minutes using the $0-1 \mathrm{GN}$ formulation and the specialized solution approach. The fourth version was by far the most difficult, involving 173 constraints, 126 zeroone variables, and 511 continuous variables. The original mixed integer formulation was run for seven hours on an IBM 4381 using MPSX and then taken off the machine to avoid further computer-run costs. At the end of the seven hours, the best (minimum cost) solution obtained had an objective function value of

$\$ 136,173,440$. We imposed a time limit of 30 minutes on the $0-1 \mathrm{GN}$ solution effort and obtained a solution that had an objective function value of $\$ 125,174,727$ : an improvement of more than $\$ 10,000,000$. As this application shows, using the netform approach can improve the solutions for problems too complex to be solved optimally (within practical time limits) by standard approaches.

In another application of integer generalized networks, Glover, Klingman, and Mote [1989] addressed the problem of de- veloping optimization models and procedures for maximizing the collective proficiency (or "readiness") levels of US Army units. The models they proposed address both equipment procurement decisions and allocation decisions. The mathematical formulations of these models constitute largescale, mixed-integer programs that potentially contain over 8,500 constraints and 150,000 discrete variables. The new approach developed for this problem may be viewed as an integration of optimization strategies and intelligent search strategies. The integer programming formulation can be given a netform representation as a discrete generalized network (Figure 7), providing a basis for identifying structure capable of being exploited both mathematically and heuristically.

The preliminary results that the authors obtained from empirical testing on randomly generated problem data are very encouraging. Prescriptions derived from the solutions indicate that the US Army may be able to improve total unit proficiency levels by as much as 25 percent, while dramatically reducing solution time (compared to the Army's current expert system, solution time will go from 17 hours to less than four minutes for a standard sized problem). Based on these results, the US Army is launching a longrange development project to implement this approach.

\section{Future Directions}

We are entering a new age of computer applications. The evolution of ideas and perspectives that has led to expert systems, knowledge engineering, object oriented programming, and intelligent work stations has underscored the fundamental role of 


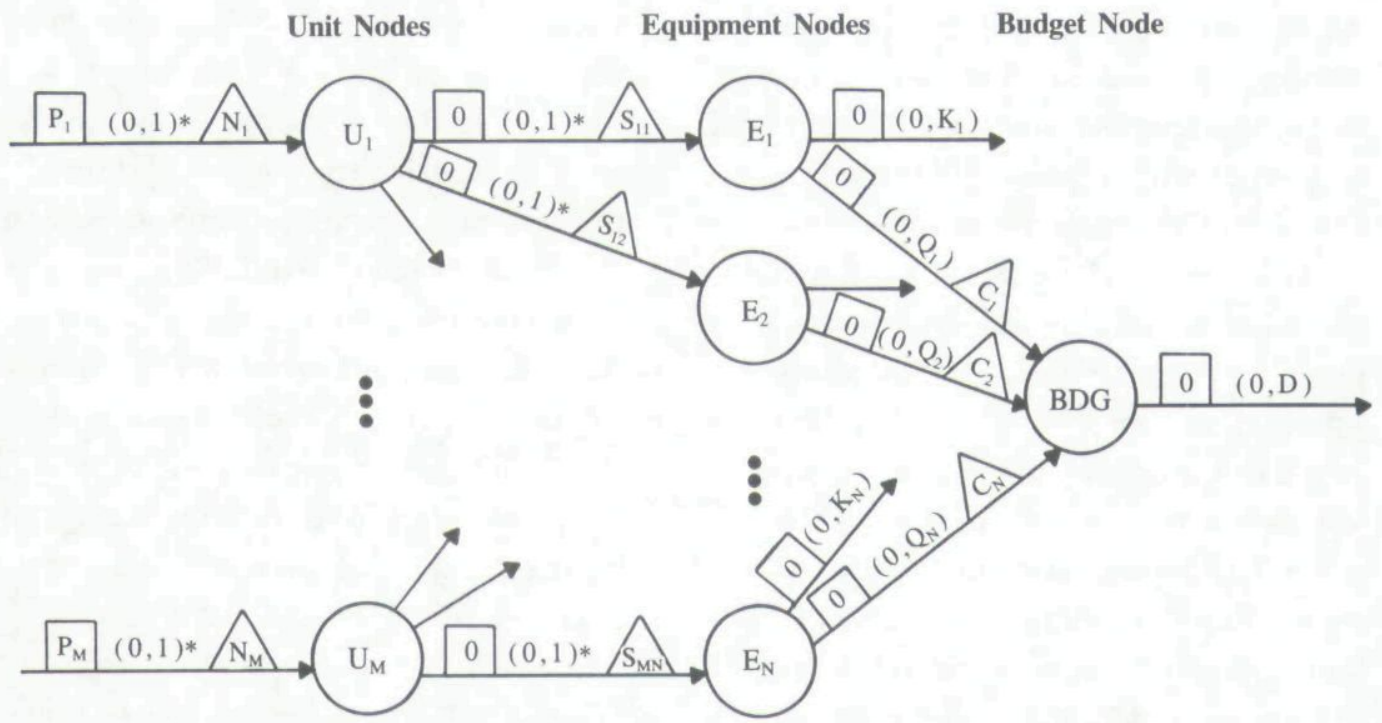

Figure 7: Mixed integer generalized network formulation for Army readiness. The arcs into nodes $U_{i}$ represent the decision to upgrade unit $i$ to its desired proficiency rating. The arc between nodes $U_{i}$ and $E_{j}$ represent whether unit $i$ is allocated equipment type $j$ to eliminate a shortage. The two arcs out of node $E_{j}$ represent the number of items of equipment allocated from inventory or procured, respectively. The coefficients are defined as follows: $P_{i}=$ positive weight assigned to unit $i$ denoting the unit's relative priority for proficiency upgrading; $N_{i}$ $=$ number of equipment categories in which unit $i$ must eliminate equipment shortages in order to receive the desired proficiency rating; $S_{i j}=$ minimum number of items of equipment type $j$ which unit $i$ must receive in order to eliminate a shortage; $C_{j}=$ unit cost to procure one item of equipment type $j ; K_{j}=$ number of items of equipment category $j$ in inventory; $Q_{j}$ $=$ upper bound on procurement quantities for equipment category $j$; and $D=$ total fiscal budget available for procurement.

representational systems in interactions between human beings and machines. Because we tend to express complex relationships by pictures and diagrams, technology is relying increasingly on pictorial representations as an indispensable element of new advances.

Netforms are being drawn into this process in a natural fashion and are currently stimulating the design of intelligent interfaces on several fronts. Netforms' legacy of successful applications of visual modeling technology, spanning diverse business, government and scientific settings, provides a background for bringing pictorial representations into human-machine interfaces to achieve practical, bottom-line benefits.

Greenberg [1987, 1988] and Murphy, Stohr and Asthana [1988] propose uses of netforms as integral parts of system diagnosis and suggest ways to create intelligent front and back ends for computer optimization software. Glover and Greenberg [1987] propose using netforms to enhance the operation of expert systems. A growing body of research is dedicated to detecting network structures that may be hidden inside more complex models [Bixby 1984; Bixby and Wagner 1985; Brown, McBride and Wood 1985; Truemper 1983]. This has led more recently to research into creating 
these and other netform structures in problem settings where they may not otherwise be found [Glover and Klingman 1988a].

Currently, Freeman et al. [1989] are developing a computer system for causal analysis called PRONET in the telecommunications industry that is an artificial intelligence (AI) tool to facilitate reasoning about complex interrelationships. The underlying constructs of this system are highly compatible with those of netforms, and they envision linking the AI features with optimization by relying more fully on netform representations in the next stage.

With these innovative developments, netforms are being used in a growing number of realms, and these uses affect how problems are translated into solvable forms and how they are conceptualized and communicated. More important, these applications are affecting the range of problems we perceive as susceptible to formulation, enlarging the domains we can represent for obtaining improved insights and solutions.

\section{Acknowledgments}

This research was supported in part by the Center for Business Decision Analysis, the Hugh Roy Cullen Centennial Chair in Business Administration, and the Office of Naval Research under contracts N0001487-K-0190 and N00014-89-J-1132. Reproduction in whole or in part is permitted for any purpose of the US Government.

\section{References}

Barr, R.; Glover, F.; and Klingman, D. 1977, "The alternating basis algorithm for assignment problems," Mathematical Programming, Vol. 13, No. 1, pp. 1-13.

Barr, R.; Glover, F.; and Klingman, D. 1979, "Enhancements of spanning tree labeling procedures for network optimization," INFOR, Vol. 17, No. 1, pp. 16-34.
Bertsekas, D. and Tseng, P. 1988, "Relaxation methods for minimum cost ordinary and generalized network flow problems," Operations Research, Vol. 36, No. 1, pp. 93-114.

Bhaumik, G. 1973, "Optimum operating policies of a water distribution system with losses," PhD diss., University of Texas at Austin.

Bixby, R. 1984, "Recent algorithms for two versions of graph realization and remarks on applications to linear programming," in Progress in Combinatorial Optimization, ed., W. R. Pulleyblank, Academic Press, pp. 39-67.

Bixby, R. and Wagner, D. 1985, "Almost lineartime algorithm for graph realization," Technical Report 85-2, Department of Mathematical Sciences, Rice University, Texas.

Bradley, G.; Brown, G.; and Graves, G. 1977, "Design and implementation of large-scale primal transshipment algorithms," Management Science, Vol. 24, No. 1, pp. 1-34.

Brown, G.; McBride, R.; and Wood, R. 1985, "Extracting embedded generalized networks from linear programming problems," Mathematical Programming, Vol. 32, No. 1, pp. 11-31.

Charnes, A. and Cooper, W. 1961, Management Models and Industrial Applications of Linear Programming, Vols. I and II, John Wiley and Sons, New York.

Collins, M.; Cooper, L.; Helgason, R.; Kennington, J.; and LeBlanc, L. 1978, "Solving the pipe network analysis problem using optimization techniques," Management Science, Vol. 24, No. 7, pp. 747-760.

Crum, R. 1976, "Cash management in the multinational firm: A constrained generalized network approach," working paper, The University of Florida.

Crum, R.; Klingman, D.; and Tavis, L. 1979, "Implementation of large-scale financial planning models: Solution efficient transformations," Journal of Financial and Quantitative Analysis, Vol. 14, No. 1, pp. 137-152.

Cunningham, W. 1979, "Theoretical properties of the network simplex method," Mathematics of Operations Research, Vol. 4, No. 2, pp. 196-208.

Dantzig, G. 1963, Linear Programming and Extensions, Princeton University Press, Princeton, New Jersey.

Evans, J. 1978, "A single commodity transfor- 
mation for certain multicommodity networks," Operations Research, Vol. 26, No. 4, pp. 673-680.

Ford, R. and Fulkerson, D. 1962, Flows in Networks, Princeton University Press, Princeton, New Jersey.

Freeman, E.; Sylvan, L.; Laufman, S.; and Blumenthal, R. 1989, "PRONET: a logicbased implementation of a generic network modeling system," Technical Report AKSR103, US West Advanced Technologies, Englewood, Colorado.

Galil, Z. and Tardos, E. 1986, "An $O\left(n^{2}(m+n\right.$ $\log n) \log n$ )min-cost flow algorithm," Proceedings of the 27th Annual Symposium on the Foundation of Computer Science, IEEE Computer Society Press, Washington, DC, pp. 136-146.

Gilliam, G. and Turner, J. 1974, "A profile analysis network model to reduce the size of microdata files," working paper, Office of Tax Analysis, Office of the Secretary of the Treasury, Washington, DC.

Gilsinn, J. and Witzgall, C. 1973, "A performance comparison of labeling algorithms for calculating shortest path trees," NBS Technical Note 772, US Department of Commerce.

Glover, F.; Glover, R.; and Martinson, F. 1984, "A netform system for resource planning in the US Bureau of Land Management," Journal of the Operational Research Society, Vol. 35 , No. 7, pp. 605-616.

Glover, F. and Greenberg, H. 1987, "Netforms provide powerful tools for enhancing operations of expert systems," Proceedings of the Rocky Mountain Conference on Artificial Intelligence, Colorado Institute for Artificial Intelligence, Boulder, Colorado, pp. 259-268.

Glover, F.; Hultz, J.; and Klingman, D. 1978, "Improved computer-based planning techniques," Part I, Interfaces, Vol. 8, No. 4, pp. $16-25$.

Glover, F.; Hultz, J.; and Klingman, D. 1979. "Improved computer-based planning techniques," Part II, Interfaces, Vol. 9, No. 4, pp. 12-20.

Glover, F.; Hultz, J.; Klingman, D.; and Stutz, J. 1978, "Generalized networks: A fundamental computer-based planning tool," Management Science, Vol. 24, No. 12, pp. 1209-1220.

Glover, F.; Karney, D.; and Klingman, D. 1972, "The augmented predecessor index method for locating stepping stone paths and assigning dual prices in distribution problems," Transportation Science, Vol. 6, No. 2, pp. 171-179.

Glover, F.; Karney, D.; and Klingman, D. 1974, "Implementation and computational study on start procedures and basis change criteria for a primal network code," Networks, Vol. 4, No. 3, pp. 191-212.

Glover, F.; Karney, D.; Klingman, D.; and Napier, A. 1974, "A computational study on start procedures, basis change criteria and solution algorithms for transportation problems," Management Science, Vol. 20, No. 5, pp. 793-819.

Glover, F. and Klingman, D. 1977, "Network application in industry and government," AIIE Transactions, Vol. 9, No. 4, pp. 363-376.

Glover, F. and Klingman, D. 1982, "Developments in computer implementation technology for network flow algorithms," INFOR, Vol. 20, No. 4, pp. 433-452.

Glover, F. and Klingman, D. 1988a, "Layering strategies for creating exploitable structure in linear and integer programs," Mathematical Programming, Vol. 40, No. 2, pp. 165-181.

Glover, F. and Klingman, D. 1988b, "A pseudopolynomial time primal simplex algorithm for minimum cost network flow problems," CBDA 154, Center for Business Decision Analysis, University of Texas at Austin.

Glover, F.; Klingman, D.; and McMillan, C. 1977, "The netform concept," Research Report CCS 281, Center for Cybernetic Studies, University of Texas at Austin.

Glover, F.; Klingman, D.; and Mote, J. 1989, "Intelligent decision system for logistical support," CBDA 157, Center for Business Decision Analysis, University of Texas at Austin.

Glover, F.; Klingman, D.; and Phillips, N. 1988, "A network-related nuclear power plant model with an intelligent branch-and-bound solution approach," CBDA 139, Center for Business Decision Analysis, University of Texas at Austin, (to appear in Annals of Operations Research).

Glover, F.; Klingman, D.; and Stutz, J. 1974, "The augmented threaded index method for network optimization," INFOR, Vol. 12, No. 3, pp. 293-298.

Glover, F. and Mulvey, J. 1980, "Equivalence of the $0-1$ integer programming problem to dis- 
crete generalized and pure networks," Operations Research, Vol. 28, No. 3, Part 2,

pp. 829-836.

Greenberg, H. 1987, "Diagnosing infeasibility for min cost network flow models; Part I: Dual infeasibility," IMA Journal of Mathematics in Management, Vol. 1, No. 1, pp. 99-110.

Greenberg, H. 1988, "Diagnosing infeasibility of min cost network flow models; Part II: Primal infeasibility," IMA Journal of Mathematics in Management, Vol. 4, No. 2, pp. 119-126.

Helgason, R.; Kennington, J.; and Lall, H. 1976, "Primal simplex network codes: State-of-theart implementation technology," Technical Report IEOR 76014, Department of Industrial Engineering and Operations Research, Southern Methodist University.

Hung, M. 1983, “A polynomial simplex method for the assignment problem," Operations Research, Vol. 31, No. 3, pp. 595-600.

Ikura, Y. and Nemhauser, G. 1986, "Computational experience with a polynomial-time dual simplex algorithm for the transportation problem," Discrete Applied Mathematics,

Vol. 13, No. 2, 3, pp. 239-248.

Jensen, P. and Barnes, W. 1980, Network Flow Programming, John Wiley and Sons, New York.

Karney, D. and Klingman, D. 1976, "Implementation and computational study on an in-core out-of-core primal network code," Operations Research, Vol. 24, No. 6, pp. 1056-1077.

Kazmersky, P. 1974, "A computer code for refueling and energy scheduling containing an evaluator of nuclear decisions for operation," PhD diss., Ohio State University.

Kennington, J. and Helgason, R. 1980, Algorithms for Network Programming, WileyInterscience, New York.

Kim, Y. 1972, "An optimal computational approach to the analysis of a generalized network of copper refining process," paper presented at the Joint ORSA/TIMS/AIIE Conference, Atlantic City, New Jersey.

Klingman, D.; Mote, J.; and Phillips, N. 1988, "A logistics planning system at W. R. Grace," Operations Research, Vol. 36, No. 6, pp. 811-822.

Klingman, D. and Phillips, N. 1984,

"Topological and computational aspects of preemptive multi-criteria military personnel assignment problems," Management Science,
Vol. 30, No. 11, pp. 1362-1375.

Klingman, D.; Phillips, N.; Steiger, D.; Wirth, R.; Padman, R.; and Krishnan, R. 1987, "An optimization-based integrated short-term refined petroleum product planning system," Management Science, Vol. 33, No. 7, pp. 813-830.

Klingman, D.; Phillips, N.; Steiger, D.; Wirth, R.; and Young, W. 1986, "The challenges and success factors in implementing an integrated petroleum products planning system for Citgo," Interfaces, Vol. 16, No. 3, pp. 1-19.

Murphy, F.; Stohr, E.; and Asthana, A. 1988, "Representation schemes for mathematical programming models," working paper, Center for Research in Information Systems, New York University.

Orlin, J. 1984, "Genuinely polynomial simplex and nonsimplex algorithms for the minimum cost flow problem," Technical Report No. 1615-84, Sloan School of Management, MIT.

Orlin, J. 1988, "A faster strongly polynomial minimum cost flow algorithm," working paper, OR 175-88, MIT.

Ross, T. and Soland, R. 1975, "A branch-andbound algorithm for the generalized assignment problem" Mathematical Programming, Vol. 8, No. 1, pp. 91-104.

Srinivasan, V. and Thompson, G. 1972, "Accelerated algorithms for labeling and relabeling of trees with applications for distribution problems," JACM, Vol. 19, No. 4, pp. 712-726.

Srinivasan, V. and Thompson, G. 1973, "Benefit-cost analysis of coding techniques for the primal transportation algorithm," JACM,

Vol. 20, No. 2, pp. 194-213.

Taha, H. 1971, Operations Research, The Macmillan Company, New York.

Tardos, E. 1985, "A strongly polynomial minimum cost circulation algorithm," Combinatorica, Vol. 5, No. 3, pp. 247-255.

Truemper, K. 1983, "How to detect hidden networks and totally unimodular subsections of linear programs," paper presented at the TIMS/ORSA Joint National Meeting, April.

Wagner, H. 1969, Principles of Operations Research, Prentice-Hall, Englewood Cliffs, New Jersey. 
Copyright 1990, by INFORMS, all rights reserved. Copyright of Interfaces is the property of INFORMS: Institute for Operations Research and its content may not be copied or emailed to multiple sites or posted to a listserv without the copyright holder's express written permission. However, users may print, download, or email articles for individual use. 